OPEN ACCESS

Edited by:

Christophe Silien,

University of Limerick, Ireland

Reviewed by:

Giju Thomas,

Vanderbilt University, United States

Sébastien Tosi,

Institute for Research in

Biomedicine, Spain

Juan M. Bueno,

University of Murcia, Spain

*Correspondence:

Giuseppe Chirico

giuseppe.chirico@mib.infn.it

Laura Sironi

laura.sironi@unimib.it

tThese authors have contributed equally to this work

Specialty section:

This article was submitted to Cancer Imaging and Image-directed Interventions,

a section of the journa

Frontiers in Oncology

Received: 05 February 2019

Accepted: 30 May 2019

Published: 19 June 2019

Citation:

Scodellaro R, Bouzin M, Mingozzi F D'Alfonso L, Granucci F, Collini M,

Chirico G and Sironi L (2019)

Whole-Section Tumor Micro-Architecture Analysis by a Two-Dimensional Phasor-Based

Approach Applied to

Polarization-Dependent Second Harmonic Imaging.

Front. Oncol. 9:527.

doi: 10.3389/fonc.2019.00527

\section{Whole-Section Tumor} Micro-Architecture Analysis by a Two-Dimensional Phasor-Based Approach Applied to Polarization-Dependent Second
Harmonic Imaging

\author{
Riccardo Scodellaro ', Margaux Bouzin ${ }^{1}$, Francesca Mingozzi ${ }^{2}$, Laura D'Alfonso ${ }^{1}$, \\ Francesca Granucci ${ }^{2}$, Maddalena Collini ${ }^{1}$, Giuseppe Chirico ${ }^{1 * t}$ and Laura Sironi ${ }^{1 * t}$ \\ ${ }^{1}$ Physics Department, Università degli Studi di Milano-Bicocca, Milan, Italy, ${ }^{2}$ Department of Biotechnology and Biosciences, \\ Università degli Studi di Milano-Bicocca, Milan, Italy
}

Second Harmonic Generation (SHG) microscopy has gained much interest in the histopathology field since it allows label-free imaging of tissues simultaneously providing information on their morphology and on the collagen microarchitecture, thereby highlighting the onset of pathologies and diseases. A wide request of image analysis tools is growing, with the aim to increase the reliability of the analysis of the huge amount of acquired data and to assist pathologists in a user-independent way during their diagnosis. In this light, we exploit here a set of phasor-parameters that, coupled to a 2-dimensional phasor-based approach ( $\mu$ MAPPS, Microscopic Multiparametric Analysis by Phasor projection of Polarization-dependent SHG signal) and a clustering algorithm, allow to automatically recover different collagen microarchitectures in the tissues extracellular matrix. The collagen fibrils microscopic parameters (orientation and anisotropy) are analyzed at a mesoscopic level by quantifying their local spatial heterogeneity in histopathology sections (few $\mathrm{mm}$ in size) from two cancer xenografts in mice, in order to maximally discriminate different collagen organizations, allowing in this case to identify the tumor area with respect to the surrounding skin tissue. We show that the "fibril entropy" parameter, which describes the tissue order on a selected spatial scale, is the most effective in enlightening the tumor edges, opening the possibility of their automatic segmentation. Our method, therefore, combined with tissue morphology information, has the potential to become a support to standard histopathology in diseases diagnosis.

Keywords: second harmonic generation, phasor approach, collagen, cancer, two-photon microscopy, label-free imaging 


\section{INTRODUCTION}

Histopathology based on excisional biopsy and tissue staining by Hematoxylin/Eosin and ImmunoHistoChemistry (HE and IHC) $(1,2)$ is the golden standard in clinical diagnostics for accurate and timely diagnosis of cancers. Fluorescence-based techniques have also been exploited to characterize the cellular and subcellular structures, both in healthy and pathological tissues. However, these techniques are characterized by some severe drawbacks: extensive tissue manipulation that can lead to artifacts, expensive and time consuming labeling protocols, lack of $3 \mathrm{D}$ sectioning, and, most important, delay in obtaining the diagnosis and interpretative variability (3-5).

In this landscape, label-free microscopy has gained increasing success due to its capability to overcome the above-mentioned issues and to aid pathologists in fast and reliable tumor diagnosis, with potentially great impact on the health of the population and the sustainability of the healthcare systems (6-8). Recently, the intrinsic fluorescence signal coming from intracellular molecules (e.g., NADH, FAD, flavoproteins, lipofuscins, or aminoacids) or tissue constituents, has been exploited to provide diagnostic information on different pathologies (911). Moreover, hyperspectral imaging which analyzes the (auto)-fluorescence or reflectance spectral profiles peculiar of each tissue, has become a promising method in diseases evaluation (12-15). Also Coherent Anti-Stokes Raman Scattering (CARS) microscopy, that provides information on the molecular composition of the sample through the Raman spectrum, has been used to identify and characterize the properties of tumors (16-18).

Furthermore, apart from the fluorescence intensity and its spectral features, fluorescence excited state lifetime (19-21) and non-linear scattering properties of the tissues, such as Polarization-resolved Second Harmonic Generation, P-SHG (2231), have been exploited. These features have been also combined with a phasor-based analysis to extract information on healthy and pathological tissue conditions (32-40) and to identify diseases such as tumor types and their development stages.

Despite the high resolution, the molecular contrast achieved and the reduced scattering, non-linear optical based techniques still suffer the disadvantage of limited penetration depth (typically $<1 \mathrm{~mm}$ ) into tissues and reduced field of view ( $\sim 500$ $\mu \mathrm{m}^{2}$ ). These limitations are at least partially overcome by optical coherence tomography (OCT) $(41,42)$ and Photo-Acustic (PA) imaging (43). OCT is a minimally invasive technique, that can be performed in real time and in situ, and that offers a high structural sensitivity, with a resolution of $1-10 \mu \mathrm{m}$ and a penetration depth of $2-3 \mathrm{~mm}$, although it provides unspecific contrast. On the other hand, PA imaging offers a higher penetration depth with respect to OCT, while it suffers from low resolution and low structural contrast.

In parallel to the efforts devoted to improve the image acquisition modes, image analysis tools that can assist pathologists in the characterization of the tissue properties are gathering increasing interest, with the aim to reach realtime automated in-vivo cancer diagnosis. User independent algorithms for the automatic extraction of disease features are attracting more and more attention due to their deep implications for resolving pathological cues and increasing the reliability of the results at reduced costs (44-49). Moreover, due to the large amount of acquired data, a dimensionality reduction for the extraction of significant and valuable features describing diseased tissues has to be faced.

By working along the above research lines, we recently developed $\mu$ MAPPS (Microscopic Multiparametric Analysis by Phasor projection of Polarization-dependent SHG signal), a $2 \mathrm{D}$ phasor analysis of the polarization dependent SHG signal collected on images (39) to provide micro-structural information (at pixel level) of the collagen architecture in the tissue extra-cellular matrix (ECM). In fact, collagen molecules suffer remodeling during pathological development that could be exploited for diagnostic purposes $(23,50,51)$. Our $2 \mathrm{D}$ phasor algorithm provides both the mean orientation angle of the fibrils $\left(\theta_{\mathrm{F}}\right)$ and the susceptibility anisotropy parameter $(\gamma$, the ratio of off-diagonal to diagonal elements of the susceptibility tensor $\stackrel{\chi}{\chi}^{(2)}$ ), converting huge optical dataset in dispersion plots in the Fourier space. In such derived space, the application of a clustering algorithm (52) allows to further reduce the dimension of the data and to recognize automatically tissutal regions sharing similar microscopic behavior without the need to resort to direct feature segmentation.

Our main aim here is to show that the $\mu$ MAPPS phasor approach can be applied to large tissue sections (few $\mathrm{mm}$ in size, comparable with histo-pathology sections) by analyzing them as separate tiles sequentially acquired on an optical microscope. In order to test the possibility to automatically discriminate tissue regions with different collagen organization in large histopathology sections, we develop a set of parameters defined in the phasor space (called hereafter p-parameters) that work on the $\theta$ and $\gamma$ distributions at a mesoscopic level $(75-150 \mu \mathrm{m}$ regions of interests instead of single pixels).

We report a proof-of-concept of the application of this method to the automatic segmentation of tumor tissues surrounded by a skin layer in histopathology sections. We performed a two levels analysis: at a first level, the tumor and skin area were manually separated by an expert and analyzed on a global scale, while in the second one our algorithm was recursively applied on sequential regions of interest (ROI) of increasing size encompassing the entire section, in order to automatically quantify the local heterogeneity of the tissues organization. As a proof of principle of the applicability of $\mu$ MAPPS to histo-pathological analysis, we investigated two cancer models, obtained from colon carcinoma CT26 cells and breast cancer $4 \mathrm{~T} 1$ cells implanted in mice.

The p-parameters whose efficacy in highlighting the tumor edges was tested here are: the mean Cluster Elements Ratio (ratio between the number of elements in each retrieved cluster and in the most populated cluster, CER), the number of clusters $\left(\mathrm{N}_{\mathrm{C}}\right)$, and the "fibril entropy" (S). This latter parameter, which describes the tissue order on a selected spatial scale, shows to be, at least at this proof-of concept stage, the most effective and significant one to automatically discriminate different collagen microarchitectures and enlighten the tumor edges (skin-tumor boundaries). 


\section{MATERIALS AND METHODS}

\section{Two-Photon Microscopy Set-Up}

The optical setup is built around a confocal scanning head (FV-300, Olympus, Japan) mounted on an upright optical microscope (BX51, Olympus, Japan) and coupled to a fs-pulsed Ti:Sa laser $(690-1,040 \mathrm{~nm}, 80 \mathrm{MHz}$ repetition rate, Mai Tai HP, Spectra Physics, CA) (53). For the SHG signal detection, an excitation wavelength of $800 \mathrm{~nm}$ was used. The backscattered SHG signal has been acquired by a photomultiplier tube (HC12502, Hamamatsu, Japan), after being collected through a high working-distance objective $(\mathrm{NA}=0.95, \mathrm{WD}=2 \mathrm{~mm}, 20 \mathrm{X}$, water immersion, XLUMPlan FI, Olympus, Japan) and filtered by a 400/20 nm band-pass filter (Chroma Inc., Brattelboro, VT, HQ400/20). The laser polarization has been controlled by a half-wavelength waveplate placed along the optical path.

\section{Image Acquisition}

Sequential images of entire tumor sections have been acquired by rotating the half-wavelength waveplate from 0 to $180^{\circ}$ in steps of $5^{\circ}$. An excitation laser power of $\mathrm{P}_{\mathrm{exc}}=50 \mathrm{~mW}$, measured before the scanning-head, has been exploited for image acquisition of the histology samples. Each image is the result of 3 Kalman average scans and has been acquired in $3.4 \mathrm{~s}$, with a field of view (FOV) dimension of $377 \times 377 \mu \mathrm{m}^{2}(512 \times 512$ pixels). Different FOVs have been stitched by means of the Stitching plugin of the Image J software (U.S. National Institute of Health, Bethesda, Maryland, USA) to obtain the mosaic of the entire section.

Both the total tumor sections or ROIs of different sizes (from $75 \times 75$ to $150 \times 150 \mu \mathrm{m}^{2}$ ) have been analyzed by means of the $\mu$ MAPPS software coupled to the cluster algorithm.

\section{Mouse Models}

All mice, inoculated with the tumor cell lines CT26 or 4T1, were $\mathrm{BALB} / \mathrm{c}$ females of 7-12 weeks of age. The mice were kept in a pathogen-free conventional animal house facility.

The animal house is run by professional employees fully equipped with state-of-the-art instrumentation in order to maintain the standard of animal welfare at the maximum levels. All mice were housed in individual, ventilated cages with $12 \mathrm{~h}$ light/dark cycles with food and water ad libitum. Experiments were performed using protocols approved by the Institutional Animal Care and Use Committee of the University of MilanoBicocca and by the Italian Ministry of Health.

\section{Cells}

The BALB/c mouse colon carcinoma CT26 (ATCC, CRL2638) or the triple negative breast cancer $4 \mathrm{~T} 1$ cell lines were cultured in IMDM-10 complete medium: IMDM, 10\% heat-inactivated FBS (EuroClone), $2 \mathrm{mM}$ l-glutamine, $100 \mathrm{U} / \mathrm{ml}$ penicillin, $100 \mu \mathrm{g} / \mathrm{ml}$ streptomycin. Cells were collected when the confluence reached the $70 \%$.

\section{Tumor Injection and Analysis}

For the CT26 and 4T1 tumor analysis, BALB/c mice were inoculated in the deep derma in the left flank with the minimal tumorigenic dose of CT26 or 4T1 tumor cells $\left(5 \times 10^{4}\right)$ at Day 0 . Explanted tumors at Day 5 were embedded in OCT freezing media (Biooptica). Sections $(5 \mu \mathrm{m})$ were cut on a Cryostat, adhered to Superfrost Plus slide (Thermo Scientific), and then imaged under the two-photon excitation microscope.

Mice subjected to tumor injection were monitored on a daily basis for signs of discomfort, including hunched posture, ruffled fur and lack of movement within the cage. The body condition score index (a qualitative assessment of an animal overall appearance based on its weight, muscle mass, and bone prominence) was used to evaluate the welfare of the mice. Mice did not present signs of distress given the short period of time between tumor injection and killing.

Three entire tumor sections from three different animals (from 100 to 300 fields of view for each section) have been analyzed for both tumor models. The method has been also applied to five different tumor sections that were acquired as a set of 6 fields of view per sample, encompassing the skintumor boundaries.

\section{IMAGE PROCESSING}

\section{$\mu$ MAPPS Analysis Method}

The $\mu$ MAPPS method has been reported in Radaelli et al. (39), while a further description of its extension to the analysis of whole histopathology sections can be found in Appendix A.

Here we considered the following microscopic theoretical model for the SHG response $I\left(\theta_{L}^{n}\right)(31,54-56)$ :

$$
\begin{aligned}
I\left(\theta_{L}^{n}\right) & =k\left\{\sin ^{2}\left[2\left(\theta_{L}^{n}-\theta_{F}\right)\right]+\left[\sin ^{2}\left(\theta_{L}^{n}-\theta_{F}\right)\right.\right. \\
& \left.\left.+\gamma \cos ^{2}\left(\theta_{L}^{n}-\theta_{F}\right)\right]^{2}\right\}
\end{aligned}
$$

where $\theta_{L}^{n}-\theta_{F}$ is the relative angle between the laser polarization and the mean collagen fibrils orientations in the image plane (see Appendix A). $\gamma=\chi_{z z z}{ }^{(2)} / \chi_{z x x}{ }^{(2)}$ is the ratio between the entries of the susceptibility tensor $\underset{=}{\chi(2)}$, and the scale factor $\mathrm{k}$ includes the absolute intensity of the SHG signal as affected by the setup parameters.

The same clustering procedure implemented in Rodriguez et al. (52) has been exploited to obtain clusters of pixels sharing similar microscopic parameters in the entire images or in regions of interest of the tumor sections. This algorithm is based on the maximum density approach and it is applied in the $\theta_{\mathrm{F}}$, $\gamma$ ) space. Only clusters characterized by a number of elements higher than a set threshold (ET), computed as a percentage of the total number of analyzed pixels, have been considered. Moreover, the distance between each element belonging to a cluster and its center must be lower than arbitrary chosen cut-off values, named $\theta_{\mathrm{C}}$ and $\gamma_{\mathrm{C}}$.

We defined the "fibril" entropy in a region of interest as:

$$
S=\frac{-\sum_{i=1}^{N} p_{i} \log p_{i}}{-\log \frac{1}{E_{C}}}=\frac{-\sum_{i=1}^{N} \frac{x_{i}}{E_{C}} \log \frac{x_{i}}{E_{C}}}{-\log \frac{1}{E_{C}}}
$$

where $p_{i}$ is the probability of occurrence of the i-th cluster measured as the ratio between the number of elements in the $\mathrm{i}$-th cluster and the total number of clustered elements $\mathrm{E}_{\mathrm{C}}$, 
and $\mathrm{N}$ is the total number of clusters. For a perfectly ordered microstructure, we expect a single cluster, $N=1$, and $\mathrm{p}_{\mathrm{i}}=$ 1 , therefore $S=0$. For a maximally disorder microstructure, instead, we expect $\mathrm{N}=\mathrm{E}_{\mathrm{C}}$, and $\mathrm{p}_{\mathrm{i}}=1 / \mathrm{N}$. In this case, we retrieve $S=1$.

\section{Software}

All the polarization-dependent analysis based on the phasor approach, the $\theta$ and $\gamma$ p-plots, and the $\theta_{\mathrm{F}}, \gamma$, clusters, CER, $\mathrm{N}_{\mathrm{C}}$, and entropy maps have been performed by means of a custom designed $\mathrm{C}++$ based software. The $\theta$ and $\gamma$ histograms have been obtained by means of the software Origin (Origin 8.5, OriginLab Corporation). All the acquired images have been visualized and linearly contrast-adjusted using ImageJ (U.S. National Institute of Health, Bethesda, Maryland, USA) or the Photoshop software.

\section{Statistical Method}

Results are expressed as mean \pm SEM. All statistical analyses were performed by GraphPad Prism Software. Means between two groups were compared with a paired two-tailed Student's $t$ test. The degree of significance was assigned as: ${ }^{*} p \leq 0.05$ and ${ }^{* *} p \leq 0.01,{ }^{* * *} p \leq 0.001$, and ${ }^{* * * *} p \leq 0.0001$. It is noteworthy that, while $p=0.01$ already accounts for a significant difference between the analysis results, some of the parameters computed on tissues of different morphologies corresponds to even larger significance, up to $p \leq 0.0001$.

\section{RESULTS}

The efficacy of $\mu$ MAPPS in characterizing the microscopic properties of the collagen organization and their spatial heterogeneity in extended tumor sections has been tested.

The microscopic $\theta_{\mathrm{F}}$ and $\gamma$ parameters, extracted pixel-bypixel by means of $\mu$ MAPPS, are here analyzed at a mesoscopic level by characterizing and quantifying their local heterogeneity on a tunable spatial scale (the ROIs dimension) with three $\mathrm{p}$ parameters, whose aim is to maximally discriminate different collagen organization within tissues. In order to select the heterogeneity level we exploit a clustering algorithm (52), defined in the $2 \mathrm{D}$ phasor space. The parameters describing the clusters properties $\left(\mathrm{N}_{\mathrm{C}}, \mathrm{CER}\right.$, and fibril entropy) can be taken as meta-features that could then be combined to conventional morphological features to support the histopathology analysis in a user-independent way.

We report in the following an example of our analysis applied to a simple, but in our opinion meaningful, model in which a tumor tissue is surrounded by a skin layer. The aim of this study is to provide a proof-of-concept of the ability of our method to distinguish among tissue areas characterized by different local properties.

The analysis of the histology section is reported at two levels: at a first level, the tumor and skin areas have been separated and globally analyzed, while the second approach relies on the sequential analysis of regions of interest encompassing the entire section.

Figure 1A shows a tumor section $(5 \mu \mathrm{m}$ thickness, $3.8 \times 2.5$ $\mathrm{mm}^{2}$, CT26 derived colon carcinoma from mice, explanted 5 days after cells inoculation), obtained as a tilescan of sequential superimposing images $\left(377 \times 377 \mu \mathrm{m}^{2}, 512 \times 512\right.$ pixels each), acquired as a function of the laser polarization.

The collagen composing the skin and the tumor fibers, revealed by the SHG signal, shows two distinct morphologies (Figure 1A). The skin is constituted by high dense basket weaves oriented collagen fibers $(57,58)$, while the tumor is characterized by wavy, thinner, longer and more sparse fibers. Each pixel has been Fourier transformed (Discrete Fourier Transform, DFT) into a point in two connected phasor plots, as described in Appendix A and in Radaelli et al. (39). The $\theta_{\mathrm{F}}$ and $\gamma$ maps related to each single acquired image have been extracted, by exploiting Equations (A3, A4), from the relative $\theta$ and $\gamma$ p-plots, and combined to obtain the resulting color-coded mosaic $\theta_{\mathrm{F}}$ and $\gamma$ maps of the entire sample (see Figures 1B,C). Figures 1D,E show the histograms of the $\theta_{\mathrm{F}}$ and $\gamma$ values (related to the tumor section in panel A), recovered from the global phasor plots in Figures 1F,G. The anisotropy parameter $\gamma$ retrieved on the entire tumor section spans a wide range [0.8-4.5] with a monomodal distribution, while the fibrils angle is characterized by a large doubly peaked distribution. An example of the results obtained for the $4 \mathrm{~T} 1$ tumors is reported in Supplementary Figure 1.

\section{Separate Analysis of Tumor and Skin Areas}

In order to highlight the global microscopic behavior of tumor and skin regions, their boundaries were manually selected by an expert, based on tissue morphology shown in $\mathrm{H} \& \mathrm{E}$ and PicroSirius Red stained sequential sections. The $\theta_{\mathrm{F}}$ and $\gamma$ maps are reported in Figures 2A-D for the skin and the tumor areas, respectively, while the corresponding $\theta_{\mathrm{F}}$ and $\gamma$ p-plots are shown in Figures 2E-H.

Moreover, while the angles show wide distributions in both cases, the $\gamma$ values lie in the range [0.8-3.5] for the tumor, and the skin shows a tail up to $\gamma \cong 4.5$. Correspondingly, the peak value obtained by a gaussian fit of the skin distribution is slightly larger, $\langle\gamma\rangle=1.83 \pm 0.02$, than that of the tumor, $\langle\gamma\rangle=$ $1.77 \pm 0.01$, with a full width at half maximum of the distribution of $\langle\text { FWHM }\rangle_{\text {skin }}=0.95 \pm 0.05$ and $\left\langle\right.$ FWHM $>_{\text {tumor }}=0.90 \pm$ 0.03 , respectively.

\section{Clustering Algorithm and $\mathrm{p}$-Parameters}

The microstructure in-homogeneity of the tumor and skin regions has been characterized by means of the number and size of the clusters, and how they scale with the choice of the cutoff conditions.

To study how the heterogeneity of the tissue microscopic properties (obtained with tight cutoff values: $\theta_{C}=5^{\circ} ; \gamma_{C}=$ 0.2 ; $\mathrm{ET}=1 \%)$ is reflected at large spatial scale, the number of clusters $\left(\mathrm{N}_{\mathrm{C}}\right)$ and the Cluster Elements Ratio (CER) distribution have been computed on the two entire regions. At this spatial scale, the skin shows a higher (microscopic) order level with respect to the tumor. In fact, by separately analyzing the skin and the tumor area, 14 and 21 clusters were retrieved, respectively (Figures 3A,B). The ratio $\mathrm{N}_{\mathrm{C}}\left(\right.$ tumor) $/ \mathrm{N}_{\mathrm{C}}$ (skin) spans the range 
A

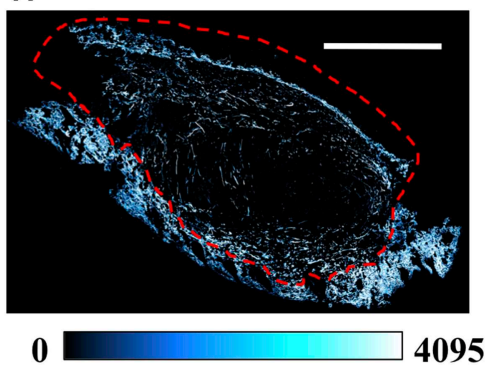

B

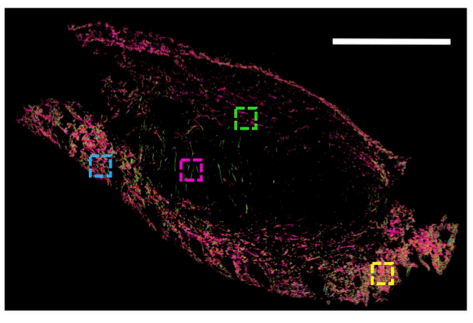

$0^{\circ}$

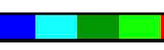

$180^{\circ}$

\section{E}
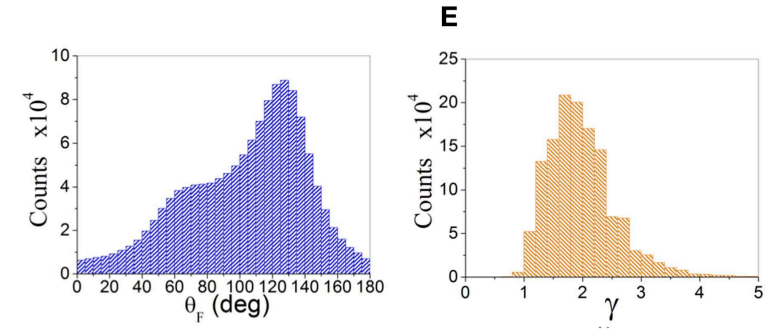

$\mathbf{F}$
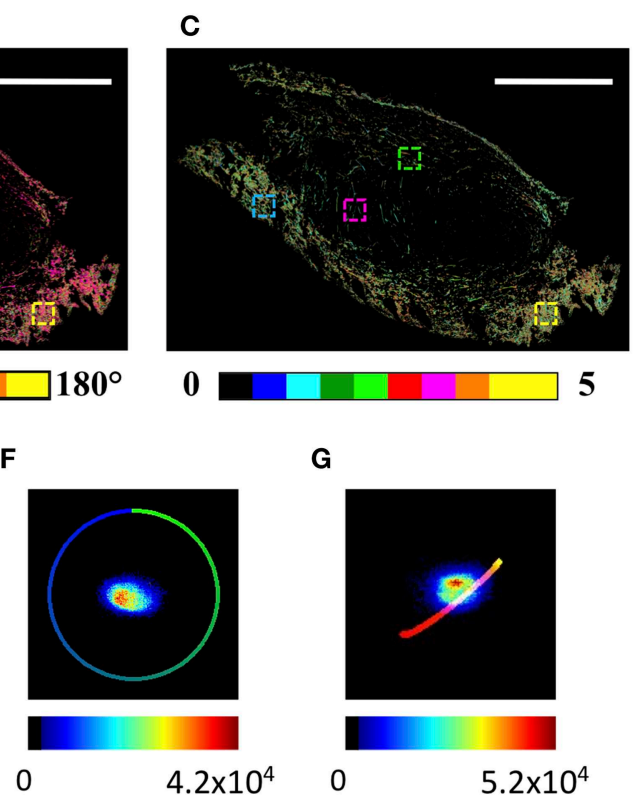

G

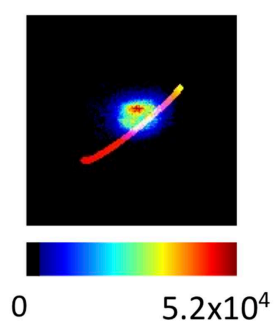

FIGURE 1 | $\mu$ MAPPS analysis of an entire tumor (CT26) section. (A) Maximum intensity projection of the mosaic reconstruction of the entire tumor section. Image size: $3.8 \times 2.5 \mathrm{~mm}^{2}$. Scale bar: $1 \mathrm{~mm}$. The dashed red line indicates the tumor-skin boundary. (B,C) Show the global $\theta$ - and $\gamma$ - maps, color-coded as in the legend. The four colored boxes show the $150 \times 150 \mu \mathrm{m}^{2}$ ROls reported in the following Figure 4. (D,E) Report the corresponding global $\theta$ - and $\gamma$ - counts histograms, while $\mathbf{( F , G ) ~ s h o w ~ t h e ~} \theta$ - and $\gamma$ - phasor plots of the entire tumor section, respectively. These results refer to the tumor section shown in $\mathbf{A}-\mathbf{C}$. The color scale encodes for the counts per phasor plot pixel. The $\theta$ reference curve in $\mathbf{( F )}$ has been obtained by simulating equation (1) within the $[0, \pi]$ angular range and $\gamma->\infty$, while the $\gamma$ reference curve in $(\mathbf{G})$ has been obtained by simulating equation (1) within the $\left[\theta_{F},\left(\theta_{F}+\pi / 2\right)\right]$ angular range while varying $\gamma$ from 0 to 10 .

[1.2-1.5] for the CT26 samples, and the range [1.25-1.35] for the $4 \mathrm{~T} 1$ tumors, and it lowers by reducing the ET value.

The CER cumulative distribution (Figures 3C,D) provides a better visualization of the sample in-homogeneity and of its dependence on the ET value. A higher number of clusters with few elements (small CER) has been retrieved for the tumor with respect to the surrounding skin, characterized instead by clusters with a more uniform population.

The tumor is characterized by a higher number of clusters with small CER with respect to the skin, although this value is strongly dependent on the selected ET. In fact, for the section reported in Figure 1A, $\sim 60 \%$ of tumor clusters have a CER value below 0.6, while this percentage is reduced to $\sim 12 \%$ for the skin (for $\theta_{\mathrm{C}}=$ $5^{\circ}, \gamma_{\mathrm{C}}=0.2$, and ET $=1 \%$, Figure 3C). However, the absolute values of the clusters number and the CER distribution values change substantially for ET $=0.5 \%$ (Figure 3D), preventing the development of a robust algorithm based on this parameter and able to discriminate between different tissue regions.

The results related to other analyzed samples are reported in Supplementary Figure 2.

In an effort to quantitatively discriminate between the two tissue regions, we moved to evaluate the "fibril" entropy ( $\mathrm{S}$, see Equation 2), which, by its definition, should provide an estimate of the local disorder degree more robust with respect to the choice of the cutoff parameters. When we assume tight clustering cutoff values, $\theta_{\mathrm{C}}=5^{\circ}, \gamma_{\mathrm{C}}=0.2$, and $\mathrm{ET}=1 \%$, in the two tissue regions, we retrieve in this sample $\sim 16 \%$ higher values of $S$ in the tumor collagen $\left(S_{\text {tumor }}=0.25\right)$ with respect to the skin $\left(S_{\text {skin }}=0.21\right)$.
Still, even if this difference is reduced to $\sim 11 \%$ for $\mathrm{ET}=0.5 \%$ $\left(\mathrm{S}_{\text {tumor }}=0.36\right.$ and $\left.\mathrm{S}_{\text {skin }}=0.32\right)$, among all the analyzed tumor sections, we retrieved a well-defined mean ratio $\mathrm{S}_{\text {tumor }} / \mathrm{S}_{\text {skin }}=$ $1.21 \pm 0.03$ for the CT26 samples $(\mathrm{ET}=1 \%)$. For $4 \mathrm{~T} 1$ tumor model, the value $S_{\text {tumor }} / S_{\text {skin }}=1.19 \pm 0.05$ has been obtained.

\section{Dependence of the Phasor Parameters on the Clustering Conditions}

A systematic investigation of the effect of the choice of the cutoff conditions $\left(\theta_{\mathrm{C}}=5^{\circ} \pm 1^{\circ}, \gamma_{\mathrm{C}}=0.20 \pm 0.02\right.$ for $\mathrm{ET}=1,0.7,0.5 \%$ ) on the evaluation of the three $\mathrm{p}$ parameters on the two tissue regions is reported in the Supplementary Materials for the tumor in Figure 1A. As shown in Supplementary Figure 3, all the three parameters can discriminate the separate collagen organization in the two macroscopic areas, with more significative results $(p<0.001)$ for $\mathrm{N}_{\mathrm{C}}$ and S. Similar results have been obtained for all the analyzed samples.

\section{Sequential Analysis on Regions of Interest With Different Sizes}

Since the boundaries between the two regions are not always sharp and the different collagen organizations can be intertwined, making difficult and user dependent an a-priori area selection, a more refined data analysis has been performed by analyzing sequential non-overlapping ROIs with a size of $75 \times 75$ and 150 $\times 150 \mu^{2}$, encompassing the entire tumor section, in order to automatically highlight different local collagen organizations 


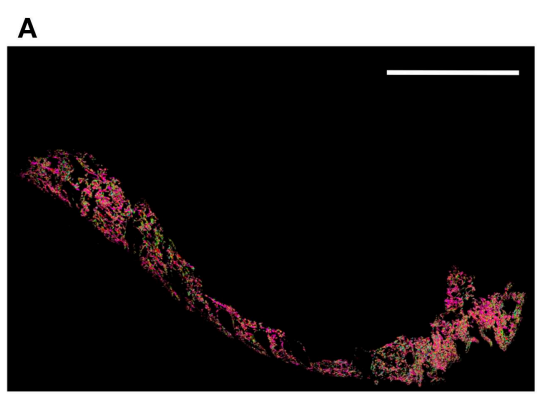

B

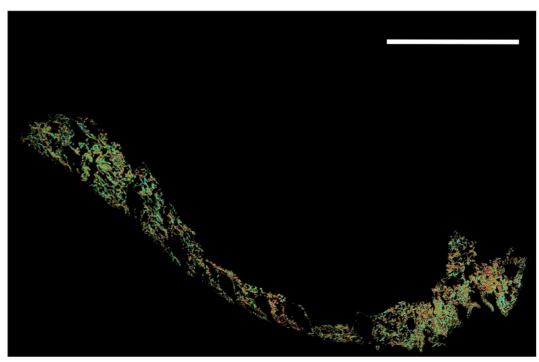

E

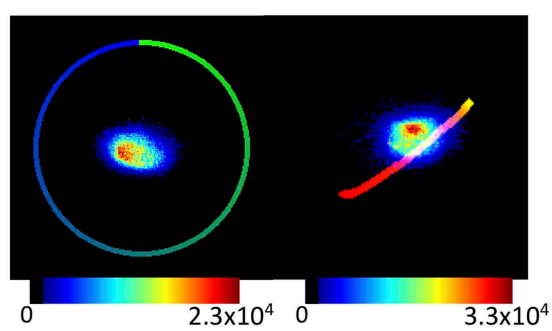

C

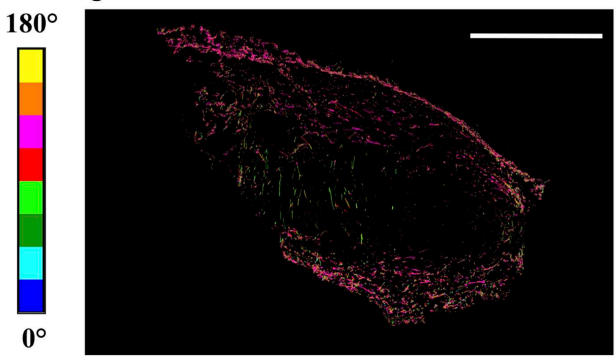

D

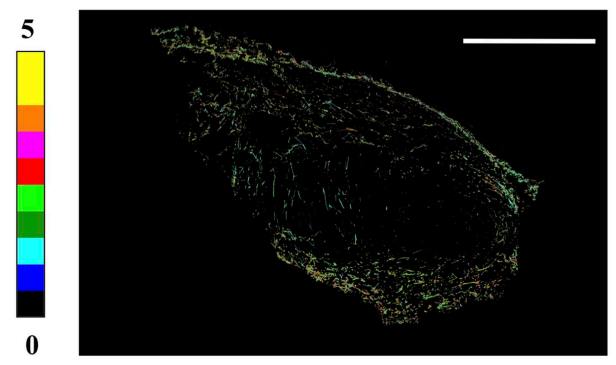

G

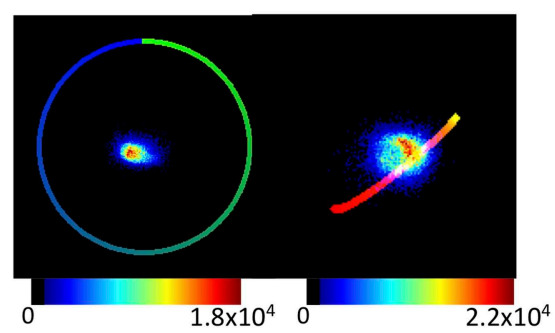

FIGURE 2 | $\mu$ MAPPS analysis of the skin and tumor regions. (A,B) show the $\theta$ - and $\gamma$ - maps, color-coded as in the legend, of the skin region, while (C,D) report those of the tumor area. Image size: $3.8 \times 2.5 \mathrm{~mm}^{2}$. Scale bar: $1 \mathrm{~mm}$. (E,F) show the $\theta$ p-plot and $\gamma \mathrm{p}$-plot of the skin region, while $(\mathbf{G}, \mathbf{H})$ report those of the tumor area. The plots were exploited to derive the $\theta$ - and $\gamma$-maps according to Equations (A3,A4). The color scale encodes for the counts per phasor plot pixel.

by means of the p-parameters. Figure 4 reports, as an example, the $\theta_{\mathrm{F}}(\mathrm{A})$ and $\gamma(\mathrm{C})$ maps for two ROIs, related to the skin and tumor regions indicated in Figure 1A, together with their distribution histograms (B,D). By taking into account all the ROIs, wider peaks for the fibrils angular distributions are mainly obtained in skin regions, while the tumor presents more variable $\gamma$ distributions with a peak in the range [1.5-2.1].

The clustering procedure has been applied to the $\theta_{\mathrm{F}}$ and $\gamma$ values retrieved in each ROI by $\mu$ MAPPS in order to compute the three p-parameters. Only ROIs where the main cluster contains at least 15 elements for the $75 \times 75 \mu \mathrm{m}^{2}$ size, and 30 for the 150 $\times 150 \mu \mathrm{m}^{2}$ size, have been considered in the analysis.

\section{CER Evaluation}

The color-coded $150 \times 150$ and $75 \times 75 \mu \mathrm{m}^{2}$ local maps have been computed while varying the cumulative CER and the ET values, by assuming the cutoff conditions $\theta_{C}=5^{\circ}, \gamma_{C}=0.2$. Although the number of clusters with a specific value of CER is the highest for ROIs belonging mainly to the skin area (coded in white color for ET $=1 \%$ and CER $=0.4$ for Figures $\mathbf{5 A}, \mathbf{B}$, and for $\mathrm{CER}=0.5$ in Figures 5C,D), this result is highly dependent on the ET and the selected CER values (as shown in Figures 5E,F for
$\mathrm{CER}=0.6$ and $\mathrm{ET}=1 \%$ ), preventing a reliable discrimination of the tumor and skin regions (see also Supplementary Figure 4).

\section{Number of Clusters Evaluation}

A more reliable result can be obtained by exploiting the number of clusters. The spatial distribution of the number of clusters is summarized in Figure 6 for low $\left(150 \times 150 \mu \mathrm{m}^{2}\right.$ A) and high $\left(75 \times 75 \mu \mathrm{m}^{2}\right.$ B) spatial sampling, assuming tight clustering conditions $\left(\theta_{C}=5^{\circ}, \gamma_{C}=0.2\right.$, ET $\left.=1 \%\right)$. A higher number of clusters is retrieved in the tumor ROIs, also when decreasing the ET (see Supplementary Figure 5). In this case, although the difference between the tumor and the skin areas is smoothed in the $\mathrm{N}_{\mathrm{C}}$ maps, it is still possible to discriminate between the two regions. Similar results have been obtained in all the analyzed tumor samples (see Supplementary Figure 6 for an example in a 4T1 tumor model sample).

\section{Fibril Entropy Evaluation}

Finally, the color-coded entropy maps, obtained with tight clustering conditions $\left(\theta_{C}=5^{\circ}, \gamma_{C}=0.2\right)$ are reported for both the CT26 and 4T1 cells derived tumors in Figures 7A,B, respectively, $\left(150 \times 150 \mu^{2}\right.$ ROI dimension with $\mathrm{ET}=$ 


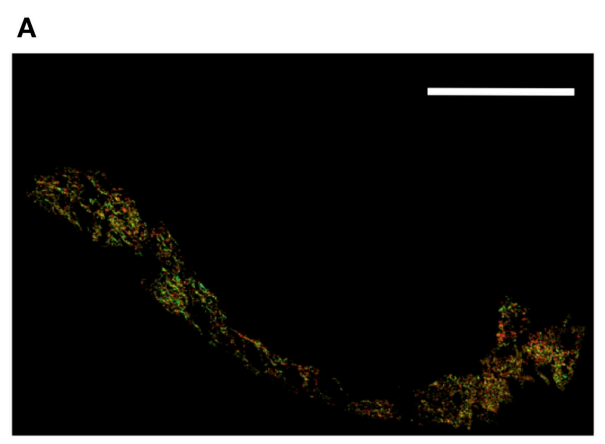

B

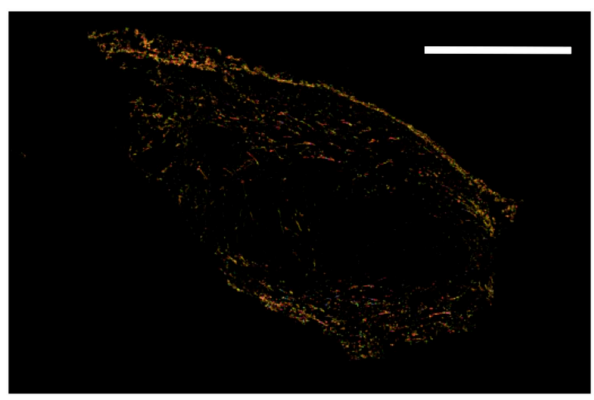

c

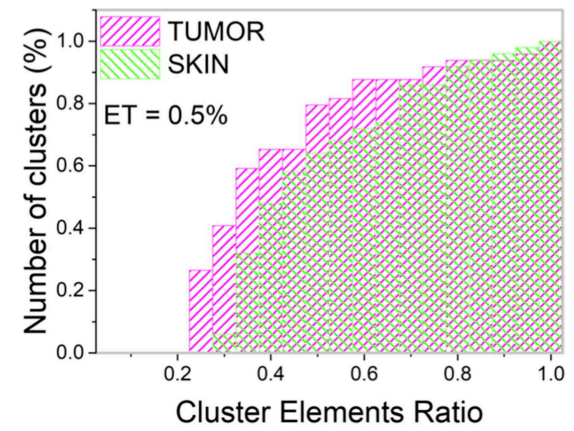

D

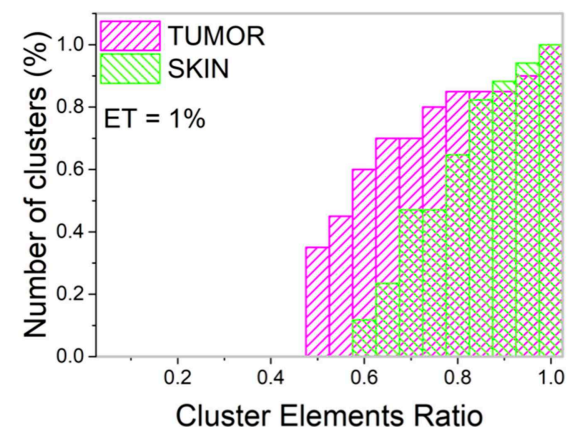

FIGURE 3 | Clustering procedure of the skin and tumor regions. (A,B) show the result of the clustering procedure applied separately to the skin (A) and the tumor (B) area by assuming $\theta_{\mathrm{C}}=5^{\circ}, \gamma_{\mathrm{C}}=0.2$, and $\mathrm{ET}=1 \%$. Image size: $3.8 \times 2.5 \mathrm{~mm}^{2}$. Scale bar: $1 \mathrm{~mm}$. Each monochromatic LUT encodes for a cluster. (C,D) show the cumulative distribution of the number of clusters (reported as percentage) as a function of the CER for the tumor (magenta) and the skin (green), related to the tumor section in Figure 1A.

1\%). In particular, from Figures $7 \mathbf{A}, \mathbf{B}$, it can be observed that $S$ is higher $(S=[0.4-0.7])$ within the tumor region with respect to the skin area and the skin/tumor edges $(S=[0.1-0.45])$. This result is maintained also at high spatial sampling. The $75 \times 75 \mu^{2}$ color-maps in fact show a similar behavior: the tumor entropy is higher with respect to the skin, with values comprised in the range [0.55-0.75] and [0.3-0.5], respectively, for $\mathrm{ET}=1 \%$ (see Supplementary Figures 7A,B). Other investigated ET conditions are reported in Supplementary Figure 8.

It is noteworthy that, in all the analyzed CT26 and 4T1 derived tumor samples, the skin and tumor areas always correspond to compact patches with a uniform value of the entropy, which is systematically $0.2-0.3$ units lower in the skin than in the tumor.

Therefore, we test the possibility to exploit the entropy parameter, fully defined in a phasor space not directly related to the sample morphology, to automatically segment regions characterized by different collagen arrangements, such as in tumor and skin. Figures 7C,D report the back-projection of the entropy information obtained for ET $=1 \%$ into the image plane for both tumor models. In the acquired intensity images we discriminated the pixels related to elements in the ROIs characterized by a "fibril entropy" above or below a selected threshold, chosen as the mean value computed in the entire section (with the clustering condition $\theta_{\mathrm{C}}=5^{\circ}, \gamma_{\mathrm{C}}=0.2$, ET $=$ $1 \%)$. We then assigned them to a monochromatic green or gray LUT in which the intensity codes for the average P-SHG signal in the pixel, and the color was assigned depending on whether the pixel entropy was below or above a selected threshold value $\mathrm{S}_{\text {th }}\left(\mathrm{S}_{\mathrm{th}}=0.4\right.$ for Figure $7 \mathrm{C} ; \mathrm{S}_{\text {th }}=0.42$ for Figure $\left.7 D\right)$. As shown in the image, the gray color highlights predominantly the skin region, with few ROIs retrieved in the tumor (mainly in the capsule region). Viceversa, the tumor is mainly enlightened by the green LUT, with some exception of the skin area at the section edge, probably due to a reduced number of clusters retrieved in ROIs encompassing the tissue-glass slide boundary. Overall, an accuracy (percentage of correctly retrieved pixels in the tumor or skin areas, obtained by comparing the number of pixels in the entropy-based segmented images with those extracted by an expert operator selection) of $(83.0 \pm 4.5) \%$ was obtained for the skin area, while the $(91.8 \pm 4.4) \%$ of the tumor area was correctly retrieved for the CT26 tumor model. For the 4T1 samples we obtained and accuracy of $(87.5 \pm 3.9) \%$ for the skin region and $(91 \pm 6) \%$ for the tumor area. Similar results can be achieved by exploiting higher spatial sampling ROIs, as shown in Supplementary Figures 7C,D. For a direct comparison of the obtained segmentation with the H\&E and PicroSirius Red stained images (exploited for the tumor-skin separation), the reader can refer to Supplementary Figure 9 (for the CT26 tumor sample 


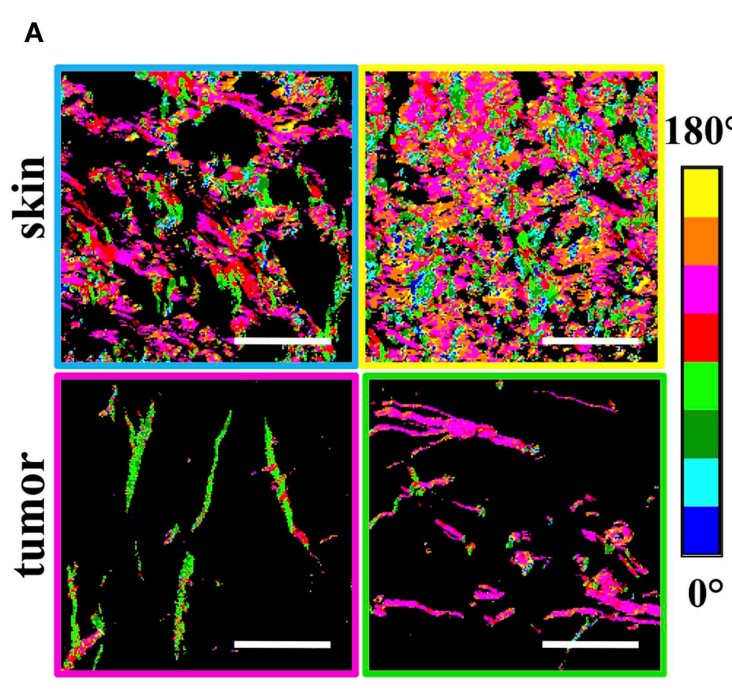

B
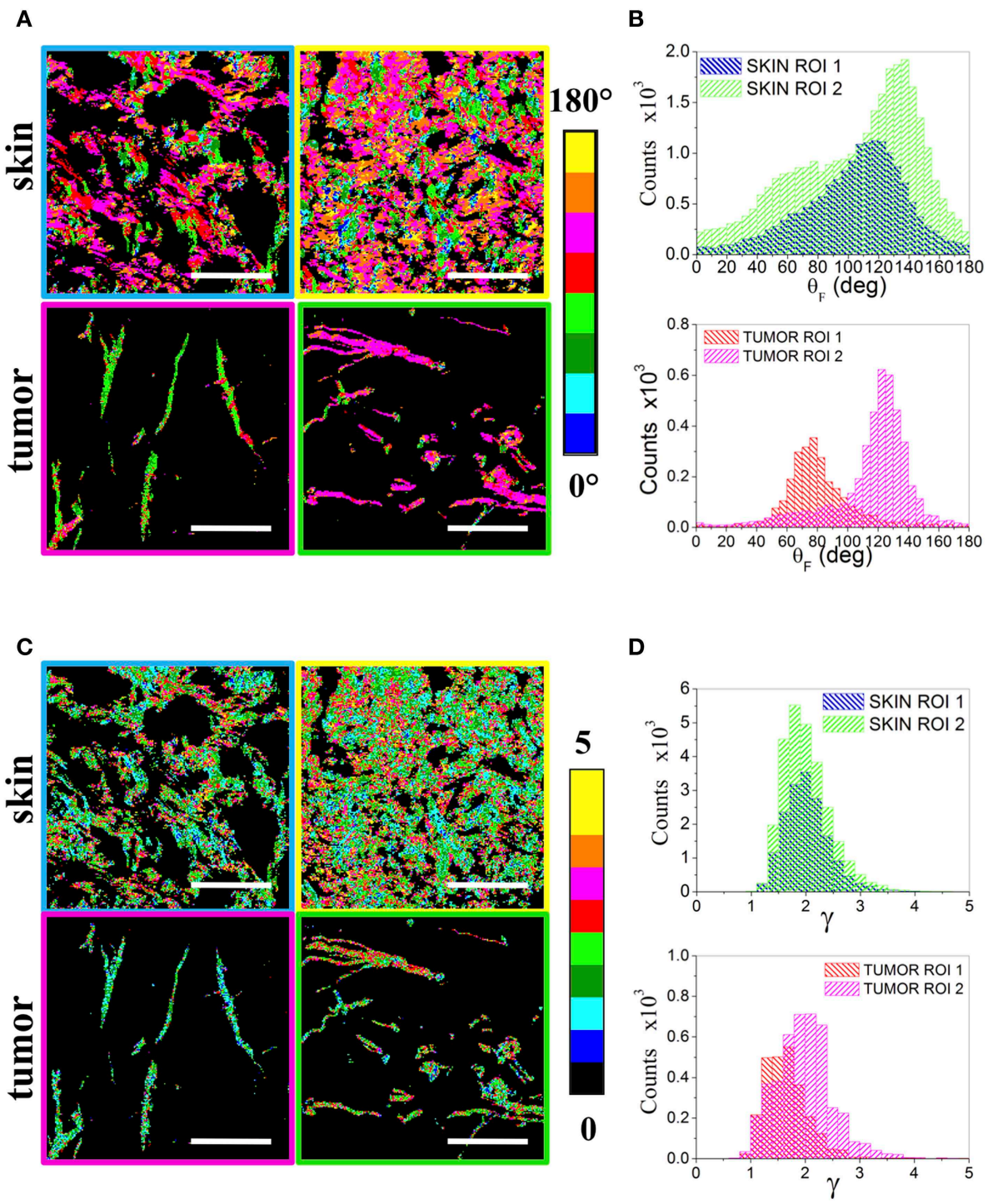

FIGURE 4 | Regions of Interest. (A) shows the $\theta_{F}$ maps of $150 \times 150 \mu \mathrm{m}^{2}$ ROls obtained from two skin (above) and two tumor (below) areas, while (B) reports the related $\theta_{F}$ distribution histograms. The corresponding $\gamma$ maps are reported in (C) for the skin (above) and the tumor (below). The ROls frames are color coded as the corresponding boxes shown in Figures 1B,C. (D) Shows the obtained $\gamma$ distribution histograms. Scale bar: $50 \mu \mathrm{m}$.

reported in Figure 1A) and Supplementary Figure 10 (for the 4T1 tumor sample shown in Supplementary Figure 1).

\section{DISCUSSION AND CONCLUSIONS}

Recently, image analysis algorithms and processing techniques attracted much attention in the histopathology field, where a rapid and precise disease diagnosis represents an increasing demand (44-49). In parallel, label-free microscopy has recently gained large interest in this field, due to its capability to image cells and tissues by exploiting the intrinsic signal of proteins and molecules, without the need of expensive and time consuming labeling protocols (6-8). However, the application of high resolution optical microscopy methods has been largely limited to demonstrative projects on small fields of view, scarcely relevant for a pathological analysis.

In this framework, we applied our phasor approach, $\mu$ MAPPS (39), to the analysis of entire fixed tumor sections, commonly exploited in histopathology, to extract the microstructural collagen fibrils angle $\left(\theta_{\mathrm{F}}\right)$ and the anisotropy $(\gamma)$ parameters. This strategy converts huge amount of optical data in dispersion plots on which clustering algorithms can be directly applied to automatically group regions that share similar properties, with the aim to highlight microstructural changes in the tissue leading to pathologies for an early tumor diagnosis and to evaluate the efficacy of a tumor treatment or rescission after surgery (59).

Here, we coupled our 2D phasor algorithm $\mu$ MAPPS to the computation of novel p-parameters in the phasor space, in 


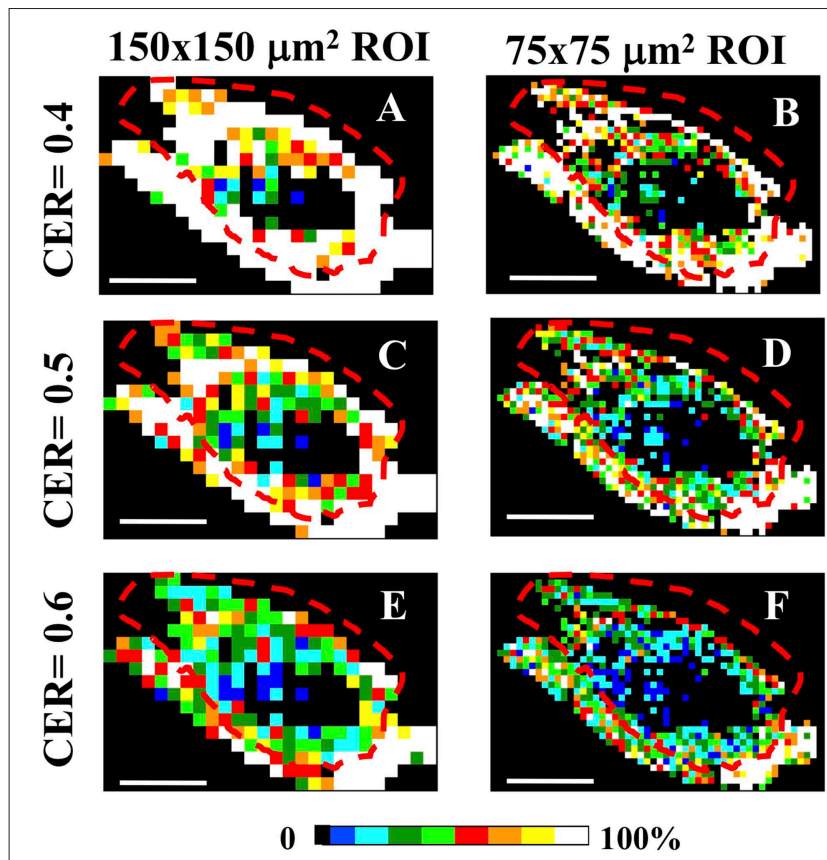

FIGURE 5 | CER analysis in Regions of Interest. (A-F) The entire tumor section has been separated in sequential non-overlapping $150 \times 150 \mu \mathrm{m}^{2} \mathbf{( A , C , E )}$ and $75 \times 75 \mu \mathrm{m}^{2} \mathbf{( B , D , F ) ~ R O I s , ~ e n c o m p a s s i n g ~ t h e ~ e n t i r e ~ t u m o r ~ s e c t i o n . ~}$ Image size: $3.8 \times 2.5 \mathrm{~mm}^{2}$. Scale bar: $1 \mathrm{~mm}$. Each $150 \times 150 \mu \mathrm{m}^{2}$ and $75 \times$ $75 \mu \mathrm{m}^{2}$ pixels $\mathrm{ROI}$ has been color-coded as in the legend to represent the percentage of clusters with a CER $=0.4(\mathbf{A}, \mathbf{B}), \mathrm{CER}=0.5 \mathbf{( C , D )}$, and CER = $0.6(\mathbf{E}, \mathbf{F})$ in each ROI. All panels share the clustering cutoff conditions $\theta_{\mathrm{C}}=$ $5^{\circ}, \gamma_{C}=0.2, E T=1 \%$. The dashed red lines indicate the tumor-skin boundary.

order to automatically recover different collagen organizations and their heterogeneity at a mesoscopic spatial scale (the ROI dimension). As a proof of concept, the method has been applied to separate the contribution of tumor collagen from skin by exploiting only microscopic structural properties retrieved in the phasor space. Among the computed parameters, the 'fibrils entropy' has been translated for the first time to the histopathology section analysis field to quantify the microscopic disorder state of clusters characterized by similar fibrils microscopic $\theta_{\mathrm{F}}$ and $\gamma$ parameters, and describe the local heterogeneity of the tissues organization.

Fixed sections in which the tumor was surrounded by a skin layer have been selected and analyzed with two parallel approaches. In the first analysis, the tumor has been manually separated from the surrounding skin edges, based on H\&E and PicroSirius Red stained sections, to highlight their different microscopic behavior. By applying $\mu$ MAPPS and the clustering algorithm on the two areas, we retrieved (for ET $=1 \%$ ) a higher number of clusters in the tumor with respect to the skin, with $\mathrm{N}_{C}$ (tumor) $/ \mathrm{N}_{\mathrm{C}}$ (skin) $=1.34 \pm 0.10$ for the CT26 tumor models and $\mathrm{N}_{\mathrm{C}}$ (tumor) $/ \mathrm{N}_{\mathrm{C}}$ (skin) $=1.30 \pm 0.03$ in the case of $4 \mathrm{~T} 1$ derived samples. Moreover, a higher entropy value has been retrieved in the tumor regions, where $S_{\text {tumor }}($ CT26) $=0.26 \pm 0.03$ and $\mathrm{S}_{\text {tumor }}(4 \mathrm{~T} 1)=0.30 \pm 0.02$ with respect to $\mathrm{S}_{\text {skin }}(\mathrm{CT} 26)=0.22$ \pm 0.03 and $\mathrm{S}_{\text {skin }}(4 \mathrm{~T} 1)=0.26 \pm 0.02$, obtained in the skin (for $\left.\theta_{\mathrm{C}}=5^{\circ}, \gamma_{\mathrm{C}}=0.2, \mathrm{ET}=1 \%\right)$. The same behavior is observed

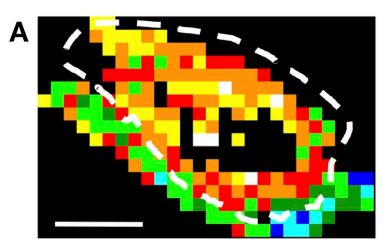

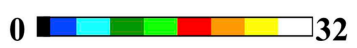

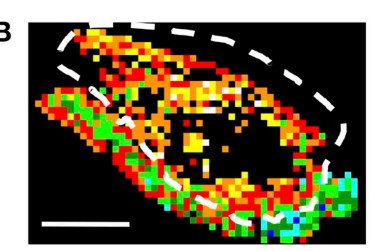

$\mathbf{0} \square+\square 37$
FIGURE 6 | Analysis of Regions of Interest: Number of Clusters $\left(\mathrm{N}_{\mathrm{C}}\right)$. (A,B) Image size: $3.8 \times 2.5 \mathrm{~mm}^{2}$. Scale bar: $1 \mathrm{~mm}$. Each $150 \times 150 \mu \mathrm{m}^{2}$ (A) and $75 \times 75 \mu \mathrm{m}^{2}$ (B) ROI has been color-coded as in the legends to represent the number of clusters retrieved in the ROls by applying the clustering cutoff conditions $\theta_{C}=5^{\circ}, \gamma_{C}=0.2$, for ET $=1 \%$. The dashed white lines indicate the tumor-skin boundary.

also for decreasing values of ET, supporting our hypothesis that this parameter can help in separating the two regions in a user independent approach, at least for the two tumor models exploited here.

A more refined and operator-independent analysis has been then performed by automatically separating the entire tumor section in $150 \times 150 \mu^{2}$ (and $75 \times 75 \mu^{2}$, see Supplementary Materials) non-overlapping windows. In this analysis, $\mu$ MAPPS and the clustering algorithm have been applied on each ROI to compute the three p-parameters: the percentage of clusters with a selected CER, the number of clusters and the "fibril entropy." We have tested the dependence of the results on the clustering procedure, the selected thresholds and the ROI dimension, and assessed to what extent we can use the pparameters to automatically highlight the different local behavior of tumor and skin collagen and which is the most effective one.

The percentage of clusters with a CER $\leq 0.6$ is an effective parameter in distinguishing the tumor from the skin when an ET $=1 \%$ is assumed. However, this difference decreases when the ET is reduced. Furthermore, tumor ROIs are characterized by a higher number of clusters with respect to the skin, with $\mathrm{N}_{\mathrm{C}}$ values comprised in the range [15-25] for the CT26 tumors and [20-25] for the $4 \mathrm{~T} 1$ samples (when $\theta_{\mathrm{C}}=5^{\circ}, \gamma_{\mathrm{C}}=0.2, \mathrm{ET}=1 \%$ ), compared to the range of $\mathrm{N}_{\mathrm{C}}$ of [15-20] in the skin. We also noticed that the entropy is $0.2-0.3$ units higher in the tumor ROIs than in the skin. Also $\mathrm{N}_{\mathrm{C}}$ and the entropy are somehow affected by the choice of the threshold ET. However, the difference of these two parameters on the two morphological area remains significative, irrespective of the ET value, and can be used to discriminate the two regions and to highlight the tumor edges. In this regard we showed (Figure 7) that the segmentation of the two regions obtained by exploiting the entropy information $(\mathrm{ET}=1 \%)$ agrees with the manual segmentation with an accuracy (computed by considering both tumor and skin areas) of $87.4 \pm 3.8 \%$ for the CT26 and $89.8 \pm 5.3 \%$ for the 4 T 1 tumor models.

We believe that these results are particularly encouraging in the direction of an automated algorithm that, by exploiting non-morphological features, will assist pathologists for a fast and reliable diagnosis. However, a number of additional tests and benchmarks are in order and some limitations need to be discussed. 

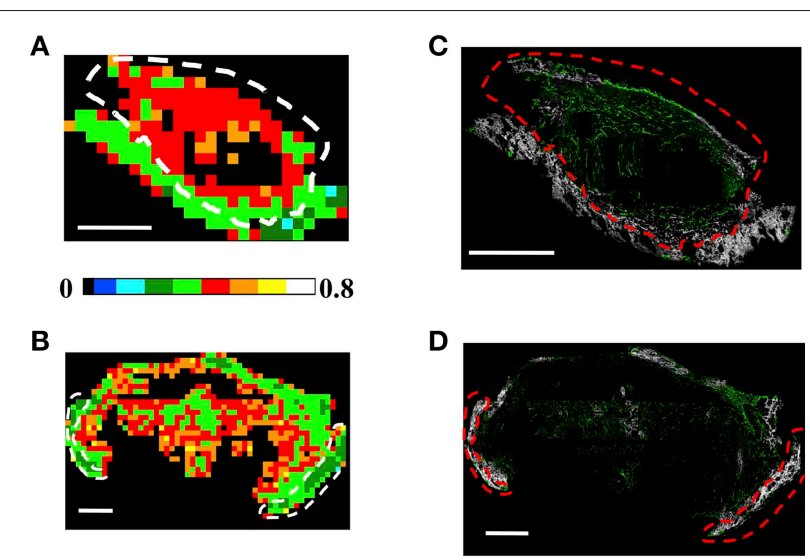

FIGURE 7 | Analysis of Regions of Interest: Fibrils Entropy S. (A,B) Each 150 $\times 150 \mu \mathrm{m}^{2} \mathbf{( A , B )} \mathrm{ROI}$ has been color-coded as in the legend to represent the retrieved entropy values in a CT26 tumor (A) and a 4T1 sample (B), by applying the clustering cutoff conditions $\theta_{C}=5^{\circ}, \gamma_{C}=0.2$, for $E T=1 \%$. Scale bar: $1 \mathrm{~mm}$. (C,D) Results of the segmentation procedure retrieved by backprojecting the entropy information into the image plane for the CT26 (C) and the 4T1 (D) tumor models. The pixels in the acquired image connected to elements in the ROls with an-entropy above a selected threshold are shown in green, while those below this threshold are colored in gray. The threshold has been chosen as the mean entropy value computed on the entire section: $\mathrm{S}_{\text {th }}$ $=0.4$ in (C) and $S_{t h}=0.42$ in (D). The dashed white and red lines indicate the tumor-skin boundary.

First of all, the computation of the $\theta_{\mathrm{F}}$ and $\gamma$ parameters was based on a specific, though widely adopted $(31,54,55)$ molecular model. Under the assumption of cylindrical and Kleinmans symmetry conditions, from the point of view of the retrieving algorithm, both the 2D and the 3D models (i.e., in plane or out of plane molecular arrangements) reduce to Equation (1) (60).

Depending on the theoretical model a-priori assumed for the specific tissue investigated, the parameter $\gamma$ assumes a different interpretation (31) since it can be related to specific molecules and fibrils arrangements (fibrils with uniform alignment, bundles of aligned or tilted fibrils). Since our aim is to discriminate between two different collagen arrangements in two different tissues in an operator-independent way, in this manuscript we did not derive microscopic geometrical parameters of the fibrils and collagen molecules inside a pixel (61-63). Instead, we introduce a statistical parameter, the "fibrils entropy," to explore the relation between both the $\theta$ and $\gamma$ values on a mesoscopic spatial scale (the ROI dimension) to automatically quantify the local heterogeneity of the tissues organization, based on the simplest model available in the literature.

However, by exploiting the a-priori knowledge of the theoretical model describing a particular tissue (31), we could further evaluate NC, CER, and S on the fibrils and molecules microscopic arrangements, retrieved from the $\gamma$ values. Moreover, refined models which take into account multiple components of the susceptibility tensor [see for example models in references $(27,28,60,61,64-67)$ ] could reveal different behavior in terms of NC, CER, and S.

For a fully exploitation in the pathology field, we need to validate our method on additional tumor models and alternative tissue sections preparations. To this aim, formalin fixed paraffin embedded sections of human tumors, already tested in P-SHG imaging (68), will be extensively investigated in our lab in order to understand if the entropy based discrimination algorithm could be advantageously applied to differentiate between tumorous and healthy regions within the same tissue type.

Regarding extension of our SHG phasor algorithm, we notice that other methods devoted to pure intensity-based image analysis, showing different collagen fibers organization among healthy and pathological tissues have been proposed. These methods quantify collagen organization from the SHG images by means of 2D Fast Fourier Transform $(69,70)$, wavelet transform (71), quantification of fiber structure and alignment (72-75), fractal analysis (76), texture analysis of SHG images using firstorder and second-order statistics (e.g., gray-level co-occurrence matrix (GLCM) (77-79), or a combination of them (80). We think that the method proposed in this manuscript could be coupled both to intensity-based image analysis algorithms and to other p-parameters, defined also in other phasor spaces, such as those based on the autofluorescence spectral and lifetime decomposition $(32,33)$, in order to expand the dictionary of features available for pathologic tissue characterization and therefore help physicians during diagnosis, especially for diseases characterized by a large heterogeneity not only between patients, but even within the same tissue specimen.

Furthermore, for an application of our 2D phasor method to thick biopsies, a fact that will dramatically simplify the tissue preparation procedure, the "fibril entropy" based threshold segmentation should be extended to a $3 \mathrm{D}$ analysis. This is particularly relevant because collagen fibers create different $3 \mathrm{D}$ ECM organization in tumors, depending on the tumor model and on the stage of development. Therefore, the extensive characterization of the collagen $3 \mathrm{D}$ structure, also from the microscopic point of view, is of fundamental importance to extract features that can be exploited for diagnosis.

As for the present application to the CT26 and 4T1 tumors inoculated in mice, we have demonstrated that our approach offers multiple advantages: it is able to perform fast microstructural analyses in entire tumor sections that could assist pathologists for a timely and precise disease diagnosis. It is costeffective since it is based on label free microscopy: exogenous fluorescent dyes are not necessary to elicit second harmonic generation signal, which instead is a non-linear coherent optical process where two incident photons of frequency $\omega$ are converted into a single photon of exactly twice the frequency and it is related to the intrinsic symmetric properties of molecules (e.g., collagen).

Our approach is based on non-linear optics scanning microscopy, which has superb capabilities to exploit endogenous optical properties of tissues (emitted intensity but also excited state lifetime or non-linear scattering polarization) and to provide optical sections (virtual non-invasive biopsies in-vivo) of the tissular architecture that can be coupled to traditional histopathology approaches with deep implications for resolving pathological cues.

In summary, the proposed $2 \mathrm{D}$ phasor approach to the labelfree second harmonic generation microscopy of collagen ECM, coupled to the definition of the "fibril entropy" parameter, is promising for the long-term goal of this project. We believe 
that automated analysis algorithms, coupled to label-free nonlinear microscopy and Fourier image processing, could represent a viable solution to assist the pathologists' interpretation of data, speeding up the analysis at reduced costs, meeting directly one of the urgent needs of our society.

\section{ETHICS STATEMENT}

Experiments were performed using protocols approved by the Institutional Animal Care and Use Committee of the University of Milano-Bicocca and by the Italian Ministry of Health.

\section{AUTHOR CONTRIBUTIONS}

LS and GC conceived the method and the experiments, and supervised the project. LS, GC, LD, and MC wrote the manuscript. RS modified the previous $\mu$ MAPPS version and

\section{REFERENCES}

1. Gurcan MN, Boucheron LE, Can A, Madabhushi A, Rajpoot NM, Yener B. Histopathological image analysis: a review. IEEE Rev Biomed Eng. (2009) 2:147-71. doi: 10.1109/RBME.2009.2034865

2. Kiernan JA. Histological and Histochemical Methods: Theory and Practice. Banbury: Scion Publishing Ltd (2015). p. 571.

3. Icha J, Weber M, Waters JC, Norden C. Phototoxicity in live fluorescence microscopy, and how to avoid it. Bioessays. (2017) 39:1700003. doi: 10.1002/bies.201700003

4. Laissue PP, Alghamdi RA, Tomancak P, Reynaud EG, Shroff H. Assessing phototoxicity in live fluorescence imaging. Nat Methods. (2017) 14:657-61. doi: $10.1038 /$ nmeth. 4344

5. Taqi SA, Sami SA, Sami LB, Zaki SA. A review of artifacts in histopathology. $J$ Oral Maxillofac Pathol. (2018) 22:279. doi: 10.4103/jomfp.JOMFP_125_15

6. Wu X, Chen G, Lu J, Zhu W, Qiu J, Chen J, et al. Label-free detection of breast masses using multiphoton microscopy. PLoS ONE. (2013) 8:e65933. doi: 10.1371/journal.pone.0065933

7. Thomas G, van Voskuilen J, Gerritsen HC, Sterenborg HJ. Advances and challenges in label-free nonlinear optical imaging using twophoton excitation fluorescence and second harmonic generation for cancer research. J Photochem Photobiol B. (2014) 141:128-38. doi: 10.1016/j.jphotobiol.2014.08.025

8. Tu H, Liu Y, Turchinovich D, Marjanovic M, Lyngsø J, Lægsgaard J, et al. Stain-free histopathology by programmable supercontinuum pulses. Nat Photonics. (2016) 10:534-40. doi: 10.1038/nphoton.2016.94

9. Zipfel WR, Williams RM, Christie R, Nikitin AY, Hyman BT, Webb WW. Live tissue intrinsic emission microscopy using multiphoton-excited native fluorescence and second harmonic generation. Proc Natl Acad Sci USA. (2003) 100:7075-80. doi: 10.1073/pnas.0832308100

10. Croce AC, Bottiroli G. Autofluorescence spectroscopy and imaging: a tool for biomedical research and diagnosis. Eur J Histochem. (2014) 58:320-37. doi: 10.4081/ejh.2014.2461

11. Bhattacharjee S, Satwaha S, Thornton K, Scholz D. Label-free imaging and optical characterization of tissues based on autofluorescence. ACS Omega. (2018) 3:5926-30. doi: 10.1021/acsomega.8b00678

12. Leavesley SJ, Walters M, Lopez C, Baker T, Favreau PF, Rich TC, et al. Hyperspectral imaging fluorescence excitation scanning for colon cancer detection. J Biomed Opt. (2016) 21:104003. doi: 10.1117/1.JBO.21.10.104003

13. Fei B, Lu G, Wang X, Zhang H, Little JV, Patel MR, et al. Label-free reflectance hyperspectral imaging for tumor margin assessment: a pilot study on surgical specimens of cancer patients. J Biomed Opt. (2017) 22:1-7. doi: 10.1117/1.JBO.22.8.086009 wrote the $\mathrm{C}++$ codes. RS, LS, MB, MC, and LD acquired and analyzed data. FM performed tumor-related preparations and experiments. LS, GC, and FG provided financial support for the experiments.

\section{FUNDING}

The research was funded by Università degli Studi di Milano-Bicocca Academic fundings 2015 to LS and GC. FG is supported by Associazione Italiana per la Ricerca sul Cancro (IG 2016Id.18842) and Fondazione Regionale per la Ricerca Biomedica (FRRB).

\section{SUPPLEMENTARY MATERIAL}

The Supplementary Material for this article can be found online at: https://www.frontiersin.org/articles/10.3389/fonc. 2019.00527/full\#supplementary-material

14. Halicek M, Lu G, Little JV, Wang X, Patel M, Griffith CC, et al. Deep convolutional neural networks for classifying head and neck cancer using hyperspectral imaging. J Biomed Opt. (2017) 22:60503. doi: 10.1117/1.JBO.22.6.060503

15. Khouj Y, Dawson J, Coad J, Vona-Davis L. Hyperspectral imaging and Kmeans classification for histologic evaluation of ductal carcinoma in situ. Front Oncol. (2018) 8:17. doi: 10.3389/fonc.2018.00017

16. Le TT, Huff TB, Cheng JX. Coherent anti-stokes raman scattering imaging of lipids in cancer metastasis. BMC Cancer. (2009) 9:42. doi: 10.1186/1471-2407-9-42

17. Legesse FB, Medyukhina A, Heuke S, Popp J. Texture analysis and classification in coherent anti-stokes raman scattering (CARS) microscopy images for automated detection of skin cancer. Comput Med Imaging Graph. (2015) 43:36-43. doi: 10.1016/j.compmedimag.2015.02.010

18. Weng S, Xu X, Li J, Wong STC. Combining deep learning and coherent antiStokes Raman scattering imaging for automated differential diagnosis of lung cancer. J Biomed Opt. (2017) 22:1-10. doi: 10.1117/1.JBO.22.10.106017

19. Provenzano PP, Eliceiri KW, Keely PJ. Multiphoton microscopy and fluorescence lifetime imaging microscopy (FLIM) to monitor metastasis and the tumor microenvironment. Clin Exp Metastasis. (2009) 26:357-70. doi: 10.1007/s10585-008-9204-0

20. Pate KT, Stringari C, Sprowl-Tanio S, Wang K, TeSlaa T, Hoverter NP, et al. Wnt signaling directs a metabolic program of glycolysis and angiogenesis in colon cancer. EMBO J. (2014) 33:1454-73. doi: 10.15252/embj.2014 88598

21. Carlson M, Watson AL, Anderson L, Largaespada DA, Provenzano PP. Multiphoton fluorescence lifetime imaging of chemotherapy distribution in solid tumors. J Biomed Opt. (2017) 22:1-9. doi: 10.1117/1.JBO.22.11.1 16010

22. Campagnola PJ, Loew LM. Second-harmonic imaging microscopy for visualizing biomolecular arrays in cells, tissues and organisms. Nat Biotechnol. (2003) 21:1356-60. doi: 10.1038/nbt894

23. Nadiarnykh O, LaComb RB, Brewer MA, Campagnola PJ. Alterations of the extracellular matrix in ovarian cancer studied by second harmonic generation imaging microscopy. BMC Cancer. (2010) 10:94. doi: 10.1186/1471-2407-10-94

24. Cicchi R, Vogler N, Kapsokalyvas D, Dietzek B, Popp J, Pavone FS From molecular structure to tissue architecture: collagen organization probed by SHG microscopy. J Biophotonics. (2013) 6:129-42. doi: 10.1002/jbio.201200092

25. Keikhosravi A, Bredfeldt JS, Sagar AK, Eliceiri KW. Second-harmonic generation imaging of cancer. Methods Cell Biol. (2014) 123:531-46. doi: 10.1016/B978-0-12-420138-5.00028-8 
26. Ávila FJ, Del Barco O, Bueno JM. Polarization dependence of aligned collagen tissues imaged with second harmonic generation microscopy. J Biomed Opt. (2015) 20:86001. doi: 10.1117/1.JBO.20.8.086001

27. Kumar R, Grønhaug KM, Romijn EI, Finnøy A, Davies CL, Drogset JO, et al. Polarization second harmonic generation microscopy provides quantitative enhanced molecular specificity for tissue diagnostics. J Biophotonics. (2015) 8:730-9. doi: 10.1002/jbio.201400086

28. Hristu R, Stanciu SG, Tranca DE, Stanciu GA. Improved quantification of collagen anisotropy with polarization-resolved second harmonic generation microscopy. J Biophotonics. (2017) 10:1171-9. doi: 10.1002/jbio.201600197

29. Mazumder N, Deka G, Wu WW, Gogoi A, Zhuo GY, Kao FJ. Polarization resolved second harmonic microscopy. Methods. (2017) 128:105-18. doi: 10.1016/j.ymeth.2017.06.012

30. Reiser K, Stoller P, Knoesen A. Three-dimensional geometry of collagenous tissues by second harmonic polarimetry. Sci Rep. (2017) 7:2642. doi: 10.1038/s41598-017-02326-7

31. Rouède D, Schaub E, Bellanger JJ, Ezan F, Scimeca JC, Baffet G, et al. Determination of extracellular matrix collagen fibril architectures and pathological remodeling by polarization dependent second harmonic microscopy. Sci Rep. (2017) 7:12197. doi: 10.1038/s41598-017-12398-0

32. Digman MA, Caiolfa VR, Zamai M, Gratton E. The phasor approach to fluorescence lifetime imaging analysis. Biophys J. (2008) 94:L14-6. doi: 10.1529/biophysj.107.120154

33. Fereidouni F, Bader AN, Gerritsen HC. Spectral phasor analysis allows rapid and reliable unmixing of fluorescence microscopy spectral images. Opt Express. (2012) 20:12729-41. doi: 10.1364/OE.20.012729

34. Ranjit S, Dvornikov A, Levi M, Furgeson S, Gratton E. Characterizing fibrosis in UUO mice model using multiparametric analysis of phasor distribution from FLIM images. Biomed Opt Express. (2016) 7:3519-30. doi: 10.1364/BOE.7.003519

35. Cutrale F, Trivedi V, Trinh LA, Chiu CL, Choi JM, Artiga MS, et al. Hyperspectral phasor analysis enables multiplexed 5D in vivo imaging. Nat Methods. (2017) 14:149-52. doi: 10.1038/nmeth.4134

36. Luo $\mathrm{T}, \mathrm{Lu} \mathrm{Y}$, Liu $\mathrm{S}$, Lin $\mathrm{D}, \mathrm{Qu}$ J. Phasor-FLIM as a screening tool for the differential diagnosis of actinic keratosis, bowen's disease, and basal cell carcinoma. Anal Chem. (2017) 89:8104-11. doi: 10.1021/acs.analchem.7b01681

37. Luo T, Lu Y, Liu S, Lin D, Qu J. Enhanced visualization of hematoxylin and eosin stained pathological characteristics by phasor approach. Anal Chem. (2017) 89:9224-31. doi: 10.1021/acs.analchem.7b01999

38. Trinh AL, Chen H, Chen Y, Hu Y, Li Z, Siegel ER, et al. Tracking functional tumor cell subpopulations of malignant glioma by phasor fluorescence lifetime imaging microscopy of NADH. Cancers. (2017) 9:E168. doi: $10.3390 /$ cancers 9120168

39. Radaelli F, D’Alfonso L, Collini M, Mingozzi F, Marongiu L, Granucci F, et al. $\mu$ MAPPS: a novel phasor approach to second harmonic analysis for in vitro-in vivo investigation of collagen microstructure. Sci Rep. (2018) 8:6314. doi: 10.1038/s41598-018-24800-6

40. Sameni S, Malacrida L, Tan Z, Digman MA. Alteration in fluidity of cell plasma membrane in huntington disease revealed by spectral phasor analysis. Sci Rep. (2018) 8:6060. doi: 10.1038/s41598-018-24031-9

41. Leitgeb RA, Baumann B. Multimodal optical medical imaging concepts based on optical coherence tomography. Front Phys. (2018) 6:114. doi: 10.3389/fphy.2018.00114

42. Wang J, Xu Y, Boppart SA. Review of optical coherence tomography in oncology. J Biomed Opt. (2017) 22:1-23. doi: 10.1117/1.JBO.22.12.121711

43. Wang LV. Multiscale photoacoustic microscopy and computed tomography. Nat Photon. (2009) 3:503-9. doi: 10.1038/nphoton.2009.157

44. Caie PD, Harrison DJ. Next-generation pathology. Methods Mol Biol. (2016) 1386:61-72. doi: 10.1007/978-1-4939-3283-2_4

45. Madabhushi A, Lee G. Image analysis and machine learning in digital pathology: challenges and opportunities. Med Image Anal. (2016) 33:170-5. doi: 10.1016/j.media.2016.06.037

46. Bengtsson E, Danielsen H, Treanor D, Gurcan MN, MacAulay C, Molnár B. Computer-aided diagnostics in digital pathology. Cytometry A. (2017) 91:551-4. doi: 10.1002/cyto.a.23151

47. Clarke EL, Treanor D. Colour in digital pathology: a review. Histopathology. (2017) 70:153-63. doi: 10.1111/his.13079
48. Hartman DJ, Pantanowitz L, McHugh JS, Piccoli AL, OLeary MJ, Lauro GR. Enterprise implementation of digital pathology: feasibility, challenges, and opportunities. J Digit Imaging. (2017) 30:555-60. doi: 10.1007/s10278-017-9946-9

49. Tizhoosh HR, Pantanowitz L. Artificial intelligence and digital pathology: challenges and opportunities. J Pathol Inform. (2018) 9:38. doi: 10.4103/jpi.jpi_53_18

50. Kaushik S, Pickup MW, Weaver VM. From transformation to metastasis: deconstructing the extracellular matrix in breast cancer. Cancer Metastasis Rev. (2016) 35:655-67. doi: 10.1007/s10555-016-9650-0

51. Bonnans C, Chou J, Werb Z. Remodelling the extracellular matrix in development and disease. Nat Rev Mol Cell Biol. (2014) 15:786-801. doi: $10.1038 / \mathrm{nrm} 3904$

52. Rodriguez A, Laio A. Machine learning. clustering by fast search and find of density peaks. Science. (2014) 344:1492-6. doi: 10.1126/science.1242072

53. Caccia M, Sironi L, Collini M, Chirico G, Zanoni I, Granucci F. Image filtering for two-photon deep imaging of lymphonodes. Eur Biophys J. (2008) 37:979-87. doi: 10.1007/s00249-008-0323-y

54. Freund I, Deutsch M. Second-harmonic microscopy of biological tissue. Opt Lett. (1986) 11:94. doi: 10.1364/OL.11.000094

55. Stoller P, Kim BM, Rubenchik AM, Reiser KM, Da Silva LB. Polarizationdependent optical second-harmonic imaging of a rat-tail tendon. J Biomed Opt. (2002) 7:205-14. doi: 10.1117/1.1431967

56. Williams RM, Zipfel WR, Webb WW. Interpreting second-harmonic generation images of collagen I fibrils. Biophys J. (2005) 88:1377-86. doi: 10.1529/biophysj.104.047308

57. Nillesen ST, Lammers G, Wismans RG, Ulrich MM, Middelkoop E, Spauwen $\mathrm{PH}$, et al. Design and in vivo evaluation of a molecularly defined acellular skin construct: reduction of early contraction and increase in early blood vessel formation. Acta Biomater. (2011) 7:1063-71. doi: 10.1016/j.actbio.2010.10.011

58. Jor JW, Parker MD, Taberner AJ, Nash MP, Nielsen PM. Computational and experimental characterization of skin mechanics: identifying current challenges and future directions. Wiley Interdiscip Rev Syst Biol Med. (2013) 5:539-56. doi: 10.1002/wsbm.1228

59. Burke K, Brown E. The use of second harmonic generation to image the extracellular matrix during tumor progression. Intravital. (2015) 3:e984509. doi: 10.4161/21659087.2014.984509

60. Psilodimitrakopoulos S, Amat-Roldan I, Loza-Alvarez P, Artigas D. Effect of molecular organization on the image histograms of polarization SHG microscopy. Biomed Opt Express. (2012) 3:2681. doi: 10.1364/BOE.3.002681

61. Kumar R, Grønhaug KM, Romijn EI, Finnøy A, Davies CL, Drogset JO, et al. Hierarchical model of fibrillar collagen organization for interpreting the second-order susceptibility tensors in biological tissue. J Biophotonics. (2015) 8:730. doi: 10.1016/j.bpj.2012.10.019

62. Campbell KR, Chaudhary R, Handel JM, Patankar MS, Campagnola PJ. Polarization-resolved second harmonic generation imaging of human ovarian cancer. J Biomed Opt. (2018) 23:1-8. doi: 10.1117/1.JBO.23.6.066501

63. Hu PS, Ghazaryan A, Hovhannisyan V, Chen SJ, Chen YF, Kim CS, et al. Imaging of biological tissues with pixel-level analysis of second-order susceptibility. J Biomed Opt. (2013) 18:31102. doi: 10.1117/1.JBO.18.3.031102

64. Su PJ, Chen WL, Chen YF, Dong CY. Determination of collagen nanostructure from second-order susceptibility tensor analysis. Biophys J. (2011) 100:2053. doi: 10.1016/j.bpj.2011.02.015

65. Psilodimitrakopoulos S, Artigas D, Soria G, Amat-Roldan I, Planas AM, LozaAlvarez P. Quantitative discrimination between endogenous SHG sources in mammalian tissue, based on their polarization response. Opt Express. (2009) 17:10168-76. doi: 10.1364/OE.17.010168

66. Tiaho F, Recher G, Rouède D. Estimation of helical angles of myosin and collagen by second harmonic generation imaging microscopy. Opt Express. (2007) 15:12286-95. doi: 10.1364/OE.15.012286

67. Su PJ, Chen WL, Hong JB, Li TH, Wu RJ, Chou CK, et al. Discrimination of collagen in normal and pathological skin dermis through secondorder susceptibility microscopy. Opt Express. (2009) 17:11161-71. doi: 10.1364/OE.17.011161

68. Blockhuys S, Agarwal NR, Hildesjö C, Jarlsfelt I, Wittung-Stafshede P, Sun XF. Second harmonic generation for collagen I characterization in rectal cancer patients with and without preoperative radiotherapy. J Biomed Opt. (2017) 22:1-6. doi: $10.1117 / 1$.JBO.22.10.106006 
69. Ghazaryan A, Tsai HF, Hayrapetyan G, Chen WL, Chen YF, Jeong MY, et al. Analysis of collagen fiber domain organization by Fourier second harmonic generation microscopy. J Biomed Opt. (2013) 18:031105. doi: 10.1117/1.JBO.18.3.031105

70. Matteini P, Ratto F, Rossi F, Cicchi R, Stringari C, Kapsokalyvas D., et al. Photothermally-induced disordered patterns of corneal collagen revealed by SHG imaging. Opt Express. (2009) 17:4868-78. doi: 10.1364/OE.17.004868

71. Liu Y, Zhu X, Huang Z, Cai J, Chen R, Xiong S, et al. Texture analysis of collagen second-harmonic generation images based on local difference local binary pattern and wavelets differentiates human skin abnormal scars from normal scars. J Biomed Opt. (2015) 20:016021. doi: 10.1117/1.JBO.20.1.0 16021

72. Chen X, Nadiarynkh O, Plotnikov S, Campagnola PJ. Second harmonic generation microscopy for quantitative analysis of collagen fibrillar structure. Nat Protoc. (2012) 7:654-69. doi: 10.1038/nprot.2012.009

73. Bredfeldt JS, Liu Y, Pehlke CA, Conklin MW, Szulczewski JM, Inman DR et al. Computational segmentation of collagen fibers from secondharmonic generation images of breast cancer. J Biomed Opt. (2014) 19:16007. doi: 10.1117/1.JBO.19.1.016007

74. Sun $\mathrm{M}$, Bloom AB, Zaman MH. Rapid quantification of $3 \mathrm{D}$ collagen fiber alignment and fiber intersection correlations with high sensitivity. PLOS ONE. (2015) 10:e0131814. doi: 10.1371/journal.pone.0131814

75. Lee B, Konen J, Wilkinson S, Marcus AI, Jiang Y. Local alignment vectors reveal cancer cell-induced ECM fiber remodeling dynamics. Sci Rep. (2017) 7:39498. doi: 10.1038/srep39498

76. Frisch KE, Duenwald-Kuehl SE, Kobayashi H, Chamberlain CS, Lakes RS, Vanderby R. Quantification of collagen organization using fractal dimensions and fourier transforms. Acta Histochem. (2012) 114:140-4. doi: 10.1016/j.acthis.2011.03.010

77. Mostaço-Guidolin LB, Ko ACT, Wang F, Xiang B, Hewko M, Tian G, et al. Collagen morphology and texture analysis: from statistics to classification. Sci Rep. (2013) 3:2190. doi: 10.1038/srep02190

78. Kirkpatrick ND, Brewer MA, Utzinger U. Endogenous optical biomarkers of ovarian cancer evaluated with multiphoton microscopy. Cancer Epidemiol Biomarkers Prev. (2007) 16:2048-57. doi: 10.1158/1055-9965.EPI-07-0009

79. Wen B, Campbell KR, Tilbury K, Nadiarnykh O, Brewer MA, Patankar $\mathrm{M}$, et al. 3D texture analysis for classification of second harmonic generation images of human ovarian cancer. Sci Rep. (2016) 6:35734. doi: 10.1038/srep35734

80. Stanciu SG, Xu S, Peng Q, Yan J, Stanciu GA, Welsch RE, et al. Experimenting liver fibrosis diagnostic by two photon excitation microscopy and Bag-ofFeatures image classification. Sci Rep. (2014) 4:4636. doi: 10.1038/srep04636

Conflict of Interest Statement: The authors declare that the research was conducted in the absence of any commercial or financial relationships that could be construed as a potential conflict of interest.

Copyright (C) 2019 Scodellaro, Bouzin, Mingozzi, D’Alfonso, Granucci, Collini, Chirico and Sironi. This is an open-access article distributed under the terms of the Creative Commons Attribution License (CC BY). The use, distribution or reproduction in other forums is permitted, provided the original author(s) and the copyright owner(s) are credited and that the original publication in this journal is cited, in accordance with accepted academic practice. No use, distribution or reproduction is permitted which does not comply with these terms. 


\section{APPENDIX A}

\section{Image Processing $\mu$ MAPPS Analysis Method}

The P-SHG signal of each pixel of a stack of images acquired as a function of the laser polarization $\theta_{\mathrm{L}}$ (Figure A1) is projected onto a first complex plane ( $\theta$ p-plot) via the first harmonic normalized Discrete Fourier Transform (DFT) applied to the $0 \leq \theta_{\mathrm{L}}<\pi$ range. In the $\theta$ p-plot, each pixel is transposed into a point, whose $\left(\mathrm{g}_{\theta}, \mathrm{s}_{\theta}\right)$ coordinates are:

$g_{\theta}=\frac{\sum_{n=0}^{N-1} I\left(\theta_{L}^{n}\right) \cos \left(\theta_{L}^{n} K_{\theta}\right)}{\sum_{n=0}^{N-1} I\left(\theta_{L}^{n}\right)} ; s_{\theta}=\frac{\sum_{n=0}^{N-1} I\left(\theta_{L}^{n}\right) \sin \left(\theta_{L}^{n} K_{\theta}\right)}{\sum_{n=0}^{N-1} I\left(\theta_{L}^{n}\right)}$

where $\left\{I\left(\theta_{L}^{n}\right)\right\}_{\mathrm{n}=0 \ldots \mathrm{N}-1}$ is the SHG intensity acquired as a function of $\theta_{\mathrm{L}}\left(0 \leq \theta_{\mathrm{L}}<3 \pi / 2\right)$ with resolution $\left.\Delta \theta ; K_{\theta}=2 \pi(\mathrm{N}) \Delta \theta\right)^{-1}$ and $\mathrm{N}=\pi / \Delta \theta$. The angular position of the points in the $\theta$ p-plot depends only on the collagen fibrils angle $\theta_{\mathrm{F}}$.

To extract the microscopic order parameter $\gamma$, a second interconnected phasor plot ( $\gamma$ p-plot) is computed by DFT of the P-SHG in the domain $\theta_{\mathrm{F}} \leq \theta_{\mathrm{L}}<\theta_{\mathrm{F}}+\pi / 2$. The coordinates $\left(\mathrm{g}_{\gamma}\right.$, $s_{\gamma}$ ) of the points in the $\gamma$ p-plot are:

$$
\begin{aligned}
& g_{\gamma}=\frac{\sum_{n=0}^{N / 2-1} I\left(\theta_{L}^{n}+\theta_{F}\right) \cos \left(\left(\theta_{L}^{n}+\theta_{F}\right) K_{\gamma}\right)}{\sum_{n=0}^{N-1} I\left(\theta_{L}^{n}+\theta_{F}\right)} ; \\
& s_{\gamma}=\frac{\sum_{n=0}^{N / 2-1} I\left(\theta_{L}^{n}+\theta_{F}\right) \sin \left(\left(\theta_{L}^{n}+\theta_{F}\right) K_{\gamma}\right)}{\sum_{n=0}^{N-1} I\left(\theta_{L}^{n}+\theta_{F}\right)}
\end{aligned}
$$

where $\mathrm{K}_{\gamma}=2 \pi\left[N\left(\Delta \theta+\theta_{F}\right)\right]^{-1}$.

To extract the $\theta_{\mathrm{F}}$ and $\gamma$ values we took advantage of two reference curves obtained for the $\theta$ p-plot and $\gamma$ p-plot by considering the microscopic theoretical model for the SHG response described by Equation (1).

The fibril angle $\theta_{\mathrm{F}}$ is half the value of the angle that the vector pointing from the center of the $\theta$ p-plot to the point $\left(g_{\theta}, s_{\theta}\right)$ makes with the phasor plot real axis and it is obtained according to the following expression:

$$
\cos \left(2 \theta_{F}\right)=\frac{g_{\theta}}{\sqrt{g_{\theta}^{2}+s_{\theta}^{2}}}
$$

The parameter $\gamma$ can been retrieved from the minimum Euclidean distance projection onto the Reference Curve (RC):

$$
\begin{aligned}
d_{e-R C} & =\sqrt{\left(g_{e}-g_{R C}\right)^{2}+\left(s_{e}-s_{R C}\right)^{2}} \\
\gamma_{e} & =\left.\gamma_{R C}\right|_{\min \left(d_{e-R C}\right)}
\end{aligned}
$$

where the minimum is obtained over the value of the $\gamma$ p-plot reference curve points, computed by exploiting the experimental sampling angle $\Delta \theta$ and $\theta_{\mathrm{F}}$ measured on the first phasor plot.

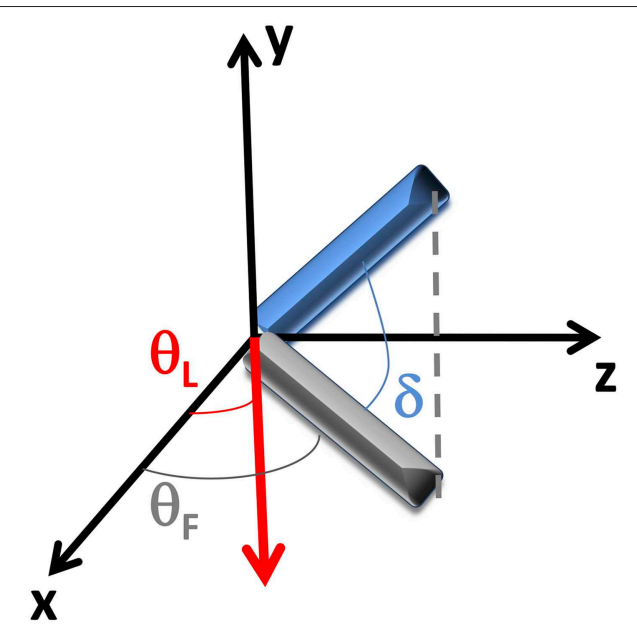

FIGURE A1 | Reference system. Coordinate system scheme: the laser is incident along the $y$-direction, while the tissue section is positioned in the $x z$ plane. The bundle of fibrils (in blue) lies out of the $x z$ plane with an angle $\delta . \theta_{F}$ represents the mean angle between the fibrils projection (in gray) into the $x z$ plane and the $\mathrm{x}$-axis, while $\theta_{\mathrm{L}}$ is the angle between the laser polarization (in red) and the $x$-axis.

The original $\mu$ MAPPS software has been modified here in order to sequentially analyze the set of images related to the entire tumor sections. The $\theta$ p-plot and $\gamma$ p-plot are computed by DFT of the P-SHG signal of each pixel in the images constituting the mosaic. The color-coded $\theta_{\mathrm{F}}$ and $\gamma$ maps are then retrieved from the phasor plots for each image by exploiting Equations (A3, A4). Finally, the $\theta_{\mathrm{F}}$ and $\gamma$ maps of the entire tumor section are obtained by mosaic combining the data related to each single image. For features of the tumor represented in two adjacent images, the mean of the $\theta_{\mathrm{F}}$ and $\gamma$ values has been computed. The software computes also the global $\theta$ p-plot and $\gamma$ p-plot of the entire tumor section, from which it is also possible to infer differences in the geometrical features related to the microscopic tissue behavior. Only P-SHG spectra above a threshold ( $\mathrm{T}_{\mathrm{NL}}$, reported in the figure captions) are analyzed in order to discard the background contribution. To further reduce the noise, a gaussian filter of the image stack acquired as function of $\theta_{\mathrm{L}}$ has been implemented before $\mu$ MAPPS analysis. Moreover, a custom implemented median filter with a $3 \times 3$ pixels mask was directly and independently applied once to the $s$ and $g$ coordinates, to reduce the dispersion of the points in the $\theta$ p-plot and $\gamma \mathrm{p}$-plot.

By means of a nearest neighbor clustering algorithm (52), applied to the two coupled phasor spaces, we could automatically group pixels characterized by $\theta_{\mathrm{F}}$ and $\gamma$ values comprised within selected cutoffs $\left(\theta_{C}\right.$ and $\left.\gamma_{C}\right)$. The total number of clustered pixels and of clusters was limited by the element threshold, ET, that is the minimum number of elements in a cluster with respect to the total number of analyzed pixels.

The software provides also the clusters, CER, $\mathrm{N}_{\mathrm{C}}$ and entropy maps of the entire tumor sections or related to the different ROI sizes. 